\title{
Quotient-3 Cordial Labelling For Cycle Related Graphs
}

\author{
Dr.P.Sumathi' ${ }^{1}$ A.Mahalakshmi ${ }^{2}$, A.Rathi ${ }^{3}$ \\ Dr.P. Sumathi ${ }^{1}$, \\ Department of Mathematics, \\ C. Kandaswami Naidu College for Men,
}

Anna nagar, Chennai 600 102. sumathipaul@yahoo.co.in

\author{
A. Mahalakshmi ${ }^{2}$, \\ Department of Mathematics, \\ Srimuthukumaran Institute of Technology, \\ Mangadu Chennai- 122. mahalakshmia.math@gmail.com \\ A. Rathi $^{3}$ \\ Department of Mathematics, \\ Dr. M.G.R. Educational and Research Institute, University, \\ Maduravoyal, Chennai 600 095. rathi.math@drmgrdu.ac.in
}

\begin{abstract}
Let $\mathrm{G}$ be a graph of order $\mathrm{p}$ and size q. Let $\mathrm{f}: \mathrm{V}(\mathrm{G}) \rightarrow \mathrm{Z}_{4}-\{0\}$ be a function. For each $\mathrm{E}(\mathrm{G})$ define $\mathrm{f}^{*}: \mathrm{E}(\mathrm{G}) \rightarrow \mathrm{Z}_{3}$ by $\mathrm{f}^{*}(\mathrm{uv})=\left\lceil\frac{f(\mathrm{u})}{f(v)}\right\rceil(\bmod 3)$ where $\mathrm{f}(\mathrm{u}) \geq \mathrm{f}(\mathrm{v})$. If the number of vertices having label $i$ and the number of vertices having label $j$ differ by maximum 1 , the number of edges having label $\mathrm{k}$ and the number of edges having label 1 differ by maximum 1 then the function $\mathrm{f}$ is said to be quotient- 3 cordial labeling of $\mathrm{G} .1 \leq \mathrm{i}, \mathrm{j} \leq 3, \mathrm{i} \neq j$ and $0 \leq \mathrm{k}, 1$ $\leq 2, k \neq 1$. Here we proved that $C_{n}$ and some cycle related graphs like $\left[C_{n} ; C_{3}\right], n=3,6,9, \ldots$, $\left(\mathrm{P}_{2} \mathrm{Umk}_{1}\right)+\mathrm{N}_{2}, \mathrm{~S}\left(\mathrm{C}_{\mathrm{n}} ; \mathrm{S}_{2}\right)$, joint sum of $\mathrm{C}_{\mathrm{n}}$ and two cycles $\mathrm{C}_{\mathrm{n}}$ having a common vertex is quotient-3 cordial.
\end{abstract}

Key words: cycle, joint sum, subdivision, quotient-3 cordial graph.

\section{Introduction}

Here the graphs considered are finite, simple, undirected and non trivial. Graph theory has a good development in the graph labeling and has a broad range of applications. Refer Gallian [2] for more information. The cordial labeling concept was first introduced by cahit [1]. The quotient-3 cordial labeling have been introduced by P. Sumathi, A. Mahalakshmi and A. Rathi found in [5-7]. They found some family of graphs are quotient-3 cordial. For notations and terminology we follow [8]. If $\mathrm{G}$ receives quotient-3 cordial labeling then $\mathrm{G}$ is called as quotient 3 cordial graph. The number of vertices having label i denotes $v_{\mathrm{f}}(\mathrm{i})$ and the number of edges having label $\mathrm{k}$ denotes $\mathrm{e}_{\mathrm{f}}(\mathrm{k}), 1 \leq \mathrm{i} \leq 3,0 \leq \mathrm{k} \leq 2$.

\section{Preliminaries}

Definition: $2.1\left[C_{n} ; C_{3}\right]$ graph is obtained by attaching the cycle $C_{3}$ with every vertex of $C_{n}$. Definition: 2.2

$\left(\mathrm{P}_{2} \mathrm{Umk}_{\mathrm{m} 1}\right)+\mathrm{N}_{2}$ graph is obtained with the vertex set $\mathrm{V}=\left\{\mathrm{z}_{1}, \mathrm{z}_{2}, \mathrm{x}_{1}, \mathrm{x}_{2} \ldots \mathrm{X}_{\mathrm{m}}\right\} \cup\left\{\mathrm{y}_{1}, \mathrm{y}_{2}\right\}$ and the edge set $\left\{\left[\left(\mathrm{y}_{1} \mathrm{z}_{1}\right),\left(\mathrm{y}_{1} \mathrm{z}_{2}\right),\left(\mathrm{y}_{2} \mathrm{z}_{1}\right),\left(\mathrm{y}_{2} \mathrm{z}_{2}\right),\left(\mathrm{z}_{1} \mathrm{z}_{2}\right)\right] \cup\left[\left(\mathrm{y}_{1} \mathrm{x}_{1}\right) \cup\left(\mathrm{y}_{2} \mathrm{x}_{\mathrm{i}}\right): 1 \leq \mathrm{i} \leq \mathrm{m}\right]\right\}$.

Definition: 2.3

A graph $\left(\mathrm{C}_{\mathrm{n}} ; \mathrm{S}_{2}\right)$ is obtained by attaching the star $\mathrm{S}_{2}$ with each vertex of a cycle $\mathrm{C}_{\mathrm{n}}$ through an edge. 
Definition: 2.4 From $G$, a graph $S(G)$ is obtained by subdividing every edge of $G$ with a new vertex is called subdivision of $\mathrm{G}$.

Definition: 2.5

A vertex of first copy of $C_{n}$ is connected with a vertex of second copy of $C_{n}$ through an edge is said to be joint sum of $C_{n}$.

\section{Main Result}

Definition: Let $G$ be a graph of order $p$ and size q. Let $f: V(G) \rightarrow Z_{4}-\{0\}$ be a function. For each $\mathrm{E}(\mathrm{G})$ define $\mathrm{f}^{*}: \mathrm{E}(\mathrm{G}) \rightarrow \mathrm{Z}_{3}$ by $\mathrm{f}^{*}(\mathrm{uv})=\left\lceil\frac{f(u)}{f(v)}\right\rceil(\bmod 3)$ where $\mathrm{f}(\mathrm{u}) \geq \mathrm{f}(\mathrm{v})$. If the number of vertices having label $\mathrm{i}$ and the number of vertices having label $\mathrm{j}$ differ by maximum 1 , the number of edges having label $\mathrm{k}$ and the number of edges having label 1 differ by maximum 1 then the function $\mathrm{f}$ is said to be quotient- 3 cordial labeling of $\mathrm{G} .1 \leq \mathrm{i}, \mathrm{j} \leq 3, \mathrm{i} \neq j$ and $0 \leq \mathrm{k}, 1$ $\leq 2, \mathrm{k} \neq 1$.

Illustration: 1 A quotient-3 cordial graph

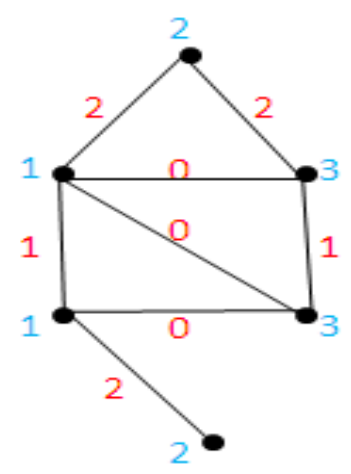

Theorem: 3.1 All cycles $C_{n}$ are quotient-3 cordial for $n \geq 4(n \neq 9,15,21, \ldots)$

Proof: Let $\mathrm{V}(\mathrm{G})=\left\{\mathrm{x}_{\mathrm{i}}: 1 \leq \mathrm{i} \leq \mathrm{n}\right\}$ and $\mathrm{E}(\mathrm{G})=\left\{\left(\mathrm{x}_{\mathrm{n}} \mathrm{x}_{1}\right),\left(\mathrm{x}_{\mathrm{i}} \mathrm{x}_{\mathrm{i}+1}\right): 1 \leq \mathrm{i} \leq \mathrm{n}-1\right\}$.

Here $|V(G)|=|E(G)|=\mathrm{n}$.

Let $\mathrm{f}: \mathrm{V}(\mathrm{G}) \rightarrow \mathrm{Z}_{4}-\{0\}$

Case (i): when $\mathrm{n} \equiv 0,1,4,5(\bmod 6)$

For all $\mathrm{i}$,

$\mathrm{f}\left(\mathrm{x}_{\mathrm{i}}\right)=1, \quad \mathrm{i} \equiv 1,2(\bmod 6)$

$\mathrm{f}\left(\mathrm{x}_{\mathrm{i}}\right)=3, \quad \mathrm{i} \equiv 0,3(\bmod 6)$

$\mathrm{f}\left(\mathrm{x}_{\mathrm{i}}\right)=2, \quad \mathrm{i} \equiv 4,5(\bmod 6)$

Case (ii): when $n \equiv 2(\bmod 6)$

Labeling of $x_{i}, 1 \leq \mathrm{i} \leq \mathrm{n}-3$ are same as in case (i).

In this case, label the vertices $\mathrm{x}_{\mathrm{n}-2}, \mathrm{x}_{\mathrm{n}-1}, \mathrm{x}_{\mathrm{n}}$ by 2,1 and 3 respectively.

\begin{tabular}{|l|l|l|l|}
\hline Nature of $\mathrm{n}$ & $\mathrm{v}_{\mathrm{f}}(1)$ & $\mathrm{v}_{\mathrm{f}}(2)$ & $\mathrm{v}_{\mathrm{f}}(3)$ \\
\hline $\mathrm{n} \equiv 0(\bmod 6)$ & $\frac{n}{3}$ & $\frac{n}{3}$ & $\frac{n}{3}$ \\
\hline $\mathrm{n} \equiv 1,4(\bmod 6)$ & $\frac{n+2}{3}$ & $\frac{n+2}{3}-1$ & $\frac{n+2}{3}-1$ \\
\hline $\mathrm{n} \equiv 2,5(\bmod 6)$ & $\frac{n+1}{3}$ & $\frac{n+1}{3}$ & $\frac{n+1}{3}-1$ \\
\hline
\end{tabular}




\begin{tabular}{|c|c|c|c|}
\hline Nature of $\mathrm{n}$ & $\mathrm{e}_{\mathrm{f}}(0)$ & $\mathrm{e}_{\mathrm{f}}(1)$ & $\mathrm{e}_{\mathrm{f}}(2)$ \\
\hline $\mathrm{n} \equiv 0(\bmod 6)$ & $\frac{n}{3}$ & $\frac{n}{3}$ & $\frac{n}{3}$ \\
\hline $\mathrm{n} \equiv 1(\bmod 6)$ & $\frac{n+2}{3}-1$ & $\frac{n+2}{3}$ & $\frac{n+2}{3}-1$ \\
\hline $\mathrm{n} \equiv 2(\bmod 6)$ & $\frac{n+1}{3}$ & $\frac{n+1}{3}$ & $\frac{n+1}{3}-1$ \\
\hline $\mathrm{n} \equiv 4(\bmod 6)$ & $\frac{n+2}{3}-1$ & $\frac{n+2}{3}-1$ & $\frac{n+2}{3}$ \\
\hline $\mathrm{n} \equiv 5(\bmod 6)$ & $\frac{n+1}{3}-1$ & $\frac{n+1}{3}$ & $\frac{n+1}{3}$ \\
\hline
\end{tabular}

Table 1

Theorem: 3.2 The graph $\left[C_{n} ; C_{3}\right]$ is quotient-3 cordial for $n=3,6,9, \ldots$

Proof: Let $V(G)=\left\{\left[x_{i}: 1 \leq i \leq n\right] \cup\left[v_{i j}: 1 \leq i \leq n, j=1,2\right]\right\}$

$\mathrm{E}(\mathrm{G})=\left\{\left[\left(\mathrm{x}_{1} \mathrm{x}_{\mathrm{n}}\right),\left(\mathrm{x}_{\mathrm{i}} \mathrm{x}_{\mathrm{i}+1}\right): 1 \leq \mathrm{i} \leq \mathrm{n}-1\right] \cup\left[\left(\mathrm{x}_{\mathrm{i}} \mathrm{v}_{\mathrm{ij}}\right): 1 \leq \mathrm{i} \leq \mathrm{n}, \mathrm{j}=1,2\right] \cup\left[\left(\mathrm{v}_{\mathrm{i} 1} \mathrm{v}_{\mathrm{i} 2}\right): 1 \leq \mathrm{i} \leq \mathrm{n}\right]\right\}$ Let $|V(\mathrm{G})|=3 n,|E(\mathrm{G})|=4 \mathrm{n}$.

Define $f: V(G) \rightarrow Z_{4}-\{0\}$

Labeling of $\mathrm{x}_{\mathrm{i}}, 1 \leq \mathrm{i} \leq \mathrm{n}$ is given below.

$\mathrm{f}\left(\mathrm{x}_{\mathrm{i}}\right)=1, \quad \mathrm{i} \equiv 1,2(\bmod 3)$

$\mathrm{f}\left(\mathrm{x}_{\mathrm{i}}\right)=3, \quad \mathrm{i} \equiv 0(\bmod 3)$

Labeling of $\mathrm{v}_{\mathrm{ij}}, 1 \leq \mathrm{i} \leq \mathrm{n}, 1 \leq \mathrm{j} \leq 2$ is given below

$\mathrm{f}\left(\mathrm{v}_{\mathrm{ij}}\right)=1, \quad \mathrm{i} \equiv 1(\bmod 3), \mathrm{j}=1$

$\mathrm{f}\left(\mathrm{v}_{\mathrm{ij}}\right)=3, \quad \mathrm{i} \equiv 1(\bmod 3), \mathrm{j}=2$

$\mathrm{f}\left(\mathrm{v}_{\mathrm{ij}}\right)=3, \quad \mathrm{i} \equiv 0(\bmod 3), \mathrm{j}=1$

$\mathrm{f}\left(\mathrm{v}_{\mathrm{ij}}\right)=2, \quad \mathrm{i} \equiv 2(\bmod 3), \mathrm{j}=1,2$

$\mathrm{f}\left(\mathrm{v}_{\mathrm{ij}}\right)=2, \quad \mathrm{i} \equiv 0(\bmod 3), \mathrm{j}=2$

For all $\mathrm{n}, \mathrm{v}_{\mathrm{f}}(1)=\mathrm{v}_{\mathrm{f}}(2)=\mathrm{v}_{\mathrm{f}}(3)=\mathrm{n}$

$$
\mathrm{e}_{\mathrm{f}}(0)=\mathrm{e}_{\mathrm{f}}(1)=\mathrm{e}_{\mathrm{f}}(2)=\frac{4 n}{3} \text {. }
$$

Theorem: 3.3 A graph $\left(P_{2} \cup n_{K}\right)+N_{2}$ is quotient-3 cordial $(n \neq 2,5,8, \ldots)$

Proof: Let $\mathrm{V}(\mathrm{G})=\left\{\mathrm{u}_{\mathrm{i}}, \mathrm{v}_{\mathrm{i}}, \mathrm{w}_{\mathrm{j}}: 1 \leq \mathrm{j} \leq \mathrm{n}, \mathrm{i}=1,2\right\}$ and

$\mathrm{E}(\mathrm{G})=\left\{\left[\left(\mathrm{u}_{1} \mathrm{u}_{2}\right),\left(\mathrm{v}_{1} \mathrm{u}_{1}\right),\left(\mathrm{v}_{1} \mathrm{u}_{2}\right),\left(\mathrm{v}_{2} \mathrm{u}_{1}\right),\left(\mathrm{v}_{2} \mathrm{u}_{2}\right)\right] \cup\left[\left(\mathrm{v}_{1} \mathrm{w}_{\mathrm{j}}\right),\left(\mathrm{v}_{2} \mathrm{w}_{\mathrm{j}}\right]: 1 \leq \mathrm{j} \leq \mathrm{n}\right]\right\}$

Let $|V(\mathrm{G})|=4+\mathrm{n},|E(\mathrm{G})|=5+2 \mathrm{n}$.

Let $\mathrm{f}: \mathrm{V}(\mathrm{G}) \rightarrow \mathrm{Z}_{4}-\{0\}$

$\mathrm{f}\left(\mathrm{u}_{1}\right)=\mathrm{f}\left(\mathrm{v}_{1}\right)=1$

$\mathrm{f}\left(\mathrm{u}_{2}\right)=\mathrm{f}\left(\mathrm{v}_{2}\right)=3$.

Label the vertices $\mathrm{w}_{\mathrm{j}}, 1 \leq \mathrm{j} \leq \mathrm{n}$ as follows

$\mathrm{f}\left(\mathrm{w}_{1}\right)=2$

When $\mathrm{n} \equiv 0,1(\bmod 3)$

For $2 \leq \mathrm{j} \leq \mathrm{n}$,

$\mathrm{f}\left(\mathrm{w}_{\mathrm{j}}\right)=1, \quad \mathrm{j} \equiv 2(\bmod 3)$

$\mathrm{f}\left(\mathrm{w}_{\mathrm{j}}\right)=2, \quad \mathrm{j} \equiv 0(\bmod 3)$

$\mathrm{f}\left(\mathrm{w}_{\mathrm{j}}\right)=3, \quad \mathrm{j} \equiv 1(\bmod 3)$ 


\begin{tabular}{|c|c|c|c|}
\hline Nature of $\mathrm{n}$ & $\mathrm{v}_{\mathrm{f}}(1)$ & $\mathrm{v}_{\mathrm{f}}(2)$ & $\mathrm{v}_{\mathrm{f}}(3)$ \\
\hline $\mathrm{n} \equiv 0(\bmod 6)$ & $\frac{n+3}{3}+1$ & $\frac{n+3}{3}$ & $\frac{n+3}{3}$ \\
\hline $\mathrm{n} \equiv 1(\bmod 6)$ & $\frac{n+2}{3}+1$ & $\frac{n+2}{3}$ & $\frac{n+2}{3}+1$ \\
\hline
\end{tabular}

\begin{tabular}{|c|c|c|c|}
\hline Nature of $\mathrm{n}$ & $\mathrm{e}_{\mathrm{f}}(0)$ & $\mathrm{e}_{\mathrm{f}}(1)$ & $\mathrm{e}_{\mathrm{f}}(2)$ \\
\hline $\mathrm{n} \equiv 0(\bmod 6)$ & $\frac{2 n+3}{3}+1$ & $\frac{2 n+3}{3}$ & $\frac{2 n+3}{3}+1$ \\
\hline $\mathrm{n} \equiv 1(\bmod 6)$ & $\frac{2 n+1}{3}+1$ & $\frac{2 n+1}{3}+1$ & $\frac{2 n+1}{3}+1$ \\
\hline
\end{tabular}

Table 2

\section{Theorem: $3.4 \mathrm{~S}\left(\mathrm{C}_{\mathrm{n}} ; \mathrm{S}_{2}\right)$ are quotient-3 cordial.}

Proof: The cycle $C_{n}$ has the vertices $u_{1}, u_{2}, u_{3} \ldots u_{n}$. Let $x_{i}$ be the vertices subdividing the edges $\left(\mathrm{u}_{\mathrm{i}} \mathrm{u}_{\mathrm{i}+1}\right)$ for all $1 \leq \mathrm{i} \leq \mathrm{n}-1$ and the vertex $\mathrm{x}_{\mathrm{n}}$ subdividing the edge $\mathrm{u}_{\mathrm{n}} \mathrm{u}_{1}$.

Let $\mathrm{V}(\mathrm{G})=\left\{\left[\mathrm{u}_{\mathrm{i}}, \mathrm{x}_{\mathrm{i}}: 1 \leq \mathrm{i} \leq \mathrm{n}\right] \cup\left[\mathrm{v}_{\mathrm{ij}}: 1 \leq \mathrm{i} \leq \mathrm{n}, \mathrm{j}=1,2\right] \cup[\right.$ wij: $\left.1 \leq \mathrm{i} \leq \mathrm{n}, \mathrm{j}=1,2]\right\}$ and $E(G)=\left\{\left[\left(u_{n} x_{n}\right),\left(x_{n} u_{1}\right),\left(u_{i} x_{i}\right),\left(x_{i} u_{i+1}\right): 1 \leq \mathrm{i} \leq n-1\right] \cup\left[\left(u_{2 i-1} v_{i j}\right): 1 \leq \mathrm{i} \leq n, j=1,2\right] \cup\left[v_{i j} w_{i j}\right):\right.$ $1 \leq \mathrm{i} \leq \mathrm{n}, \mathrm{j}=1,2]\}$

Let $|V(\mathrm{G})|=|E(\mathrm{G})|=6$.

Define $f: V(G) \rightarrow Z_{4}-\{0\}$ by

For all $\mathrm{i}, 1 \leq \mathrm{i} \leq \mathrm{n}$ and $\mathrm{j}=1,2$

$\mathrm{f}\left(\mathrm{u}_{\mathrm{i}}\right)=\mathrm{f}\left(\mathrm{x}_{\mathrm{i}}\right)=3$

$\mathrm{f}\left(\mathrm{v}_{\mathrm{ij}}\right)=1$

$\mathrm{f}\left(\mathrm{w}_{\mathrm{ij}}\right)=2$

For all $\mathrm{n}, \quad \mathrm{v}_{\mathrm{f}}(1)=\mathrm{v}_{\mathrm{f}}(2)=\mathrm{v}_{\mathrm{f}}(3)=2 \mathrm{n}$

$$
\mathrm{e}_{\mathrm{f}}(0)=\mathrm{e}_{\mathrm{f}}(1)=\mathrm{e}_{\mathrm{f}}(2)=2 \mathrm{n} \text {. }
$$

Theorem: 3.5 Joint sum of $C_{n}$ are quotient 3 cordial.

Proof: The first cycle $C_{n}$ has the vertices $u_{1}, u_{2}, \ldots, u_{n}$ and an another cycle $C_{n}$ has the vertices $\mathrm{u}_{\mathrm{n}+1}, \mathrm{u}_{\mathrm{n}+2}, \ldots, \mathrm{u}_{2 \mathrm{n}}$.

Let $V(\mathrm{G})=\left\{\left[\mathrm{u}_{\mathrm{i}}: 1 \leq \mathrm{i} \leq 2 \mathrm{n}\right]\right\}$.

$\mathrm{E}(\mathrm{G})=\left\{\left[\left(\mathrm{u}_{1} \mathrm{u}_{\mathrm{n}}\right),\left(\mathrm{u}_{\mathrm{i}} \mathrm{u}_{\mathrm{i}+1}\right): 1 \leq \mathrm{i} \leq \mathrm{n}-1\right] \cup\left[\left(\mathrm{u}_{1} \mathrm{u}_{\mathrm{n}+1}\right)\right] \cup\left[\left(\mathrm{u}_{\mathrm{i}} \mathrm{u}_{\mathrm{i}+1}\right),\left(\mathrm{u}_{\mathrm{n}+1} \mathrm{u}_{2 \mathrm{n}}\right): \mathrm{n}+1 \leq \mathrm{i} \leq 2 \mathrm{n}-1\right]\right\}$

Let $|V(\mathrm{G})|=2 n$ and $|E(\mathrm{G})|=1+2 \mathrm{n}$

Let $\mathrm{f}: \mathrm{V}(\mathrm{G}) \rightarrow \mathrm{Z}_{4}-\{0\}$

Case (i): when $\mathrm{n} \equiv 0,2,3,5(\bmod 6)$

For $1 \leq \mathrm{i} \leq 2 \mathrm{n}$

$\mathrm{f}\left(\mathrm{u}_{\mathrm{i}}\right)=1, \quad \mathrm{i} \equiv 1,2(\bmod 6)$

$\mathrm{f}\left(\mathrm{u}_{\mathrm{i}}\right)=3, \quad \mathrm{i} \equiv 0,3(\bmod 6)$

$\mathrm{f}\left(\mathrm{u}_{\mathrm{i}}\right)=2, \quad \mathrm{i} \equiv 4,5(\bmod 6)$

Case (ii): when $\mathrm{n} \equiv 1(\bmod 6)$

For $1 \leq \mathrm{i} \leq \mathrm{n}$

$\mathrm{f}\left(\mathrm{u}_{\mathrm{i}}\right)=2, \quad \mathrm{i} \equiv 1,2(\bmod 6)$

$\mathrm{f}\left(\mathrm{u}_{\mathrm{i}}\right)=3, \quad \mathrm{i} \equiv 0,3(\bmod 6)$

$\mathrm{f}\left(\mathrm{u}_{\mathrm{i}}\right)=1, \quad \mathrm{i} \equiv 4,5(\bmod 6)$

For $\mathrm{n}+1 \leq \mathrm{i} \leq 2 \mathrm{n}$ 
$\mathrm{f}\left(\mathrm{u}_{\mathrm{i}}\right)=3, \quad \mathrm{i} \equiv 1,4(\bmod 6)$

$\mathrm{f}\left(\mathrm{u}_{\mathrm{i}}\right)=1, \quad \mathrm{i} \equiv 2,3(\bmod 6)$

$\mathrm{f}\left(\mathrm{u}_{\mathrm{i}}\right)=2, \quad \mathrm{i} \equiv 0,5(\bmod 6)$

In this case after labeling all the vertices interchange the label of $u_{n+3}$ and $u_{n+4}$.

Case (iii): when $\mathrm{n} \equiv 4(\bmod 6)$

Labeling of the vertices $\mathrm{u}_{\mathrm{i}}, 1 \leq \mathrm{i} \leq 2 \mathrm{n}-1[\mathrm{i} \neq \mathrm{n}]$ are same as in Case (i)

Here $\mathrm{f}\left(\mathrm{u}_{\mathrm{n}}\right)=3, \mathrm{f}\left(\mathrm{u}_{2 \mathrm{n}}\right)=2$.

\begin{tabular}{|c|c|c|c|}
\hline Nature of $\mathrm{n}$ & $\mathrm{v}_{\mathrm{f}}(1)$ & $\mathrm{v}_{\mathrm{f}}(2)$ & $\mathrm{v}_{\mathrm{f}}(3)$ \\
\hline $\mathrm{n} \equiv 0,3(\bmod 6)$ & $\frac{2 n}{3}$ & $\frac{2 n}{3}$ & $\frac{2 n}{3}$ \\
\hline $\mathrm{n} \equiv 1(\bmod 6)$ & $\left(\frac{2 n-2}{3}\right)$ & $\left(\frac{2 n+1}{3}\right)$ & $\left(\frac{2 n+1}{3}\right)$ \\
\hline $\mathrm{n} \equiv 2(\bmod 6)$ & $\left(\frac{2 n+2}{3}\right)$ & $\left(\frac{2 n-1}{3}\right)$ & $\left(\frac{2 n-1}{3}\right)$ \\
\hline $\mathrm{n} \equiv 4(\bmod 6)$ & $\left(\frac{2 n+1}{3}\right)$ & $\left(\frac{2 n-2}{3}\right)$ & $\left(\frac{2 n+1}{3}\right)$ \\
\hline $\mathrm{n} \equiv 5(\bmod 6)$ & $\left(\frac{2 n+2}{3}\right)$ & $\left(\frac{2 n-1}{3}\right)$ & $\left(\frac{2 n-1}{3}\right)$ \\
\hline
\end{tabular}

\begin{tabular}{|c|c|c|c|}
\hline Nature of $\mathrm{n}$ & $\mathrm{e}_{\mathrm{f}}(0)$ & $\mathrm{e}_{\mathrm{f}}(1)$ & $\mathrm{e}_{\mathrm{f}}(2)$ \\
\hline $\mathrm{n} \equiv 0(\bmod 6)$ & $\frac{2 n}{3}$ & $\frac{2 n}{3}+1$ & $\frac{2 n}{3}$ \\
\hline $\mathrm{n} \equiv 1,4(\bmod 6)$ & $\left(\frac{2 n+1}{3}\right)$ & $\left(\frac{2 n+1}{3}\right)$ & $\left(\frac{2 n+1}{3}\right)$ \\
\hline $\mathrm{n} \equiv 2(\bmod 6)$ & $\left(\frac{2 n-1}{3}\right)$ & $\left(\frac{2 n+2}{3}\right)$ & $\left(\frac{2 n+2}{3}\right)$ \\
\hline $\mathrm{n} \equiv 3(\bmod 6)$ & $\frac{2 n}{3}$ & $\frac{2 n}{3}$ & $\frac{2 n}{3}+1$ \\
\hline $\mathrm{n} \equiv 5(\bmod 6)$ & $\left(\frac{2 n+2}{3}\right)$ & $\left(\frac{2 n-1}{3}\right)$ & $\left(\frac{2 n+2}{3}\right)$ \\
\hline
\end{tabular}

Table 3

Theorem: 3.6 Two copies of cycle $C_{n}$ having a common vertex is quotient-3 cordial.

Proof: Let $V(G)=\left\{\left[u_{\mathrm{i}}: 1 \leq \mathrm{i} \leq 2 \mathrm{n}-1\right]\right\}$ and

$\mathrm{E}(\mathrm{G})=\left\{\left[\left(\mathrm{u}_{1} \mathrm{u}_{\mathrm{n}}\right),\left(\mathrm{u}_{\mathrm{i}} \mathrm{u}_{\mathrm{i}+1}\right): 1 \leq \mathrm{i} \leq \mathrm{n}-1\right] \cup\left[\left(\mathrm{u}_{1} \mathrm{u}_{\mathrm{n}+1}\right),\left(\mathrm{u}_{1} \mathrm{u}_{2 \mathrm{n}-1}\right),\left(\mathrm{u}_{\mathrm{i}} \mathrm{u}_{\mathrm{i}+1}\right): \mathrm{n}+1 \leq \mathrm{i} \leq 2 \mathrm{n}-2\right]\right\}$

Let $|V(\mathrm{G})|=2 n-1$ and $|E(\mathrm{G})|=2 \mathrm{n}$

Let $\mathrm{f}: \mathrm{V}(\mathrm{G}) \rightarrow \mathrm{Z}_{4}-\{0\}$

Case (i): When $\mathrm{n} \equiv 0(\bmod 6)$

$\mathrm{f}\left(\mathrm{u}_{2 \mathrm{n}-1}\right)=3$.

For $1 \leq \mathrm{i} \leq 2 \mathrm{n}-2$

$\mathrm{f}\left(\mathrm{u}_{\mathrm{i}}\right)=1, \quad \mathrm{i} \equiv 1,2(\bmod 6)$

$\mathrm{f}\left(\mathrm{u}_{\mathrm{i}}\right)=3, \quad \mathrm{i} \equiv 0,3(\bmod 6)$

$\mathrm{f}\left(\mathrm{u}_{\mathrm{i}}\right)=2, \quad \mathrm{i} \equiv 4,5(\bmod 6)$

Case (ii): when $\mathrm{n} \equiv 1(\bmod 6)$

$\mathrm{f}\left(\mathrm{u}_{2 \mathrm{n}-3}\right)=2$

$\mathrm{f}\left(\mathrm{u}_{2 \mathrm{n}-2}\right)=1$

$\mathrm{f}\left(\mathrm{u}_{2 \mathrm{n}-1}\right)=3$

For $1 \leq \mathrm{i} \leq 2 \mathrm{n}-4$

$\mathrm{f}\left(\mathrm{u}_{\mathrm{i}}\right)=1, \quad \mathrm{i} \equiv 0,1(\bmod 6)$

$\mathrm{f}\left(\mathrm{u}_{\mathrm{i}}\right)=3, \quad \mathrm{i} \equiv 2,5(\bmod 6)$ 
$\mathrm{f}\left(\mathrm{u}_{\mathrm{i}}\right)=2, \quad \mathrm{i} \equiv 3,4(\bmod 6)$

Case (iii): when $n \equiv 2(\bmod 6)$

$\mathrm{f}\left(\mathrm{u}_{2 \mathrm{n}-2}\right)=2$.

Labeling of the vertices $u_{i}, 1 \leq i \leq 2 n-1(i \neq 2 n-2)$ are same as in case (i).

Case (iv): when $n \equiv 3(\bmod 6)$

$\mathrm{f}\left(\mathrm{u}_{2 \mathrm{n}-1}\right)=2$.

Labeling of the vertices $\mathrm{u}_{\mathrm{i}}, 1 \leq \mathrm{i} \leq 2 \mathrm{n}-2$ are same as in case (i).

Case $(v)$ : when $n \equiv 4(\bmod 6)$

Labeling of the vertices $u_{i}, 1 \leq i \leq n-1$ are same as in case (i) and $f\left(u_{n}\right)=3$.

Labeling of the vertices $u_{i}, n+1 \leq i \leq 2 n-1$ is given below.

For $1 \leq \mathrm{i} \leq 2 \mathrm{n}-1$

$\mathrm{f}\left(\mathrm{u}_{\mathrm{i}}\right)=2, \quad \mathrm{i} \equiv 1,2(\bmod 6)$

$\mathrm{f}\left(\mathrm{u}_{\mathrm{i}}\right)=3, \quad \mathrm{i} \equiv 0,3(\bmod 6)$

$\mathrm{f}\left(\mathrm{u}_{\mathrm{i}}\right)=1, \quad \mathrm{i} \equiv 4,5(\bmod 6)$

Case (vi): when $n \equiv 5(\bmod 6)$

$\mathrm{f}\left(\mathrm{u}_{2 \mathrm{n}-3}\right)=3, \mathrm{f}\left(\mathrm{u}_{2 \mathrm{n}-2}\right)=1, \mathrm{f}\left(\mathrm{u}_{2 \mathrm{n}-1}\right)=2$.

Labeling of the vertices $u_{i}, 1 \leq i \leq 2 n-4$ are same as in case (i).

\begin{tabular}{|c|c|c|c|}
\hline Nature of $\mathrm{n}$ & $\mathrm{v}_{\mathrm{f}}(1)$ & $\mathrm{v}_{\mathrm{f}}(2)$ & $\mathrm{v}_{\mathrm{f}}(3)$ \\
\hline $\mathrm{n} \equiv 0(\bmod 6)$ & $\frac{2 n}{3}$ & $\frac{2 n}{3}-1$ & $\frac{2 n}{3}$ \\
\hline $\mathrm{n} \equiv 1(\bmod 6)$ & $\left(\frac{2 n-2}{3}\right)$ & $\left(\frac{2 n+1}{3}\right)$ & $\left(\frac{2 n-2}{3}\right)$ \\
\hline $\mathrm{n} \equiv 2,5(\bmod 6)$ & $\left(\frac{2 n-1}{3}\right)$ & $\left(\frac{2 n-1}{3}\right)$ & $\left(\frac{2 n-1}{3}\right)$ \\
\hline $\mathrm{n} \equiv 3(\bmod 6)$ & $\frac{2 n}{3}$ & $\frac{2 n}{3}$ & $\frac{2 n}{3}-1$ \\
\hline $\mathrm{n} \equiv 4(\bmod 6)$ & $\left(\frac{2 n-2}{3}\right)$ & $\left(\frac{2 n-2}{3}\right)$ & $\left(\frac{2 n+1}{3}\right)$ \\
\hline
\end{tabular}

\begin{tabular}{|c|c|c|c|}
\hline Nature of $\mathrm{n}$ & $\mathrm{e}_{\mathrm{f}}(0)$ & $\mathrm{e}_{\mathrm{f}}(1)$ & $\mathrm{e}_{\mathrm{f}}(2)$ \\
\hline $\mathrm{n} \equiv 0,3(\bmod 6)$ & $\frac{2 n}{3}$ & $\frac{2 n}{3}$ & $\frac{2 n}{3}$ \\
\hline $\mathrm{n} \equiv 1(\bmod 6)$ & $\left(\frac{2 n+1}{3}\right)$ & $\left(\frac{2 n+1}{3}\right)$ & $\left(\frac{2 n-2}{3}\right)$ \\
\hline $\mathrm{n} \equiv 2(\bmod 6)$ & $\left(\frac{2 n-1}{3}\right)$ & $\left(\frac{2 n-1}{3}\right)$ & $\left(\frac{2 n+2}{3}\right)$ \\
\hline $\mathrm{n} \equiv 4(\bmod 6)$ & $\left(\frac{2 n+1}{3}\right)$ & $\left(\frac{2 n+1}{3}\right)$ & $\left(\frac{2 n-2}{3}\right)$ \\
\hline $\mathrm{n} \equiv 5(\bmod 6)$ & $\left(\frac{2 n-1}{3}\right)$ & $\left(\frac{2 n-1}{3}\right)$ & $\left(\frac{2 n+2}{3}\right)$ \\
\hline
\end{tabular}

Table 4 


\section{Conclusion}

In this paper, a quotient-3 cordial for some standard graphs has been found. The quotient-3 cordial labeling of some more graphs and graph families shall be explored in future.

\section{References}

[1] Joseph A. Gallian. A Dynamic survey of Graph Labeling, Nineteenth edition, December 23, 2016

[2] Cahit I. Cordial Graphs: A weaker version of Graceful and Harmonious graphs, Arts combin., 23 (1987), 201-207.

[3] S K Vaidya and N H Shah "Some New Families of Prime Cordial Graphs", Journal of Mathematics Research, Vol. 3, No. 4; November 2011.

[4] S K Vaidya and Lekha Bijukumar "Some New Families of E-Cordial Graphs", journal of Mathematics Research, Vol. 3, No. 4; November 2011.

[5] Sumathi P, Mahalakshmi A and Rathi A Quotient-3 Cordial Labeling for Star Related Graphs, Global Journal of Pure and Applied Mathematics, ISSN 0973-1768 Volume 13, Number 7 (2017), pp.3909-3918.

[6] Sumathi P, Mahalakshmi A and Rathi A Quotient-3 Cordial Labeling for path related graphs part-II Communicated on February 2017 and accepted June 2017 ARPN Journal of Engineering and Applied Sciences.

[7] Sumathi P, Mahalakshmi A and Rathi A Quotient-3 Cordial Labeling for path related graphs part-I Communicated on June 2017 to International Journal of Pure and Applied Mathematics.

[8] F. Harary, Graph Theory, Narosa Publishing House Reading, New Delhi ,1988. 